\title{
Switching off Electrodes in Cochlear Implants - Causes and Consequences
}

\author{
Ricardo Caiado ${ }^{1 *}$, Jorge Humberto ${ }^{1}$, Joana Pires ${ }^{1}$, Joao Eloi ${ }^{1}$, Jorge Quadros ${ }^{1}$, Luís Silva ${ }^{1}$ and \\ Pedro Tome ${ }^{1}$ \\ ${ }^{1}$ Department of Ear, Nose and Throat, Centro Hospitalar e Universitario de Coimbra, Portugal
}

Received: January 3, 2017; Accepted: January 23, 2017; Published: February 2, 2017

*Corresponding author: Dr. Ricardo Caiado, Rua Princesa Cindazunda №109, Rc B,Coimbra, Portugal, Tel:+351239400450; E-mail: ricardocaiado86@gmail.com

\begin{abstract}
Introduction: With the evolution of cochlear implantation techniques and the results achieved, the implantion indications increased. The increase of implanted patients also increases the possibility of complications related to surgical technique and the proper performance of the implanted material. The solution regarding some of these complications may be to switch off electrodes.
\end{abstract}

Objectives: To assess the causes that led to the need to switch off electrodes and its consequences in hearing performance.

Methods: Review of all cases of adult patients implanted in the Unit for Cochlear Implantation in Coimbra Hospital and University Center in which was necessary to swicth off electrodes, their causes and consequences in terms of hearing performance. We used the SPSS 23 , with T-test for paired samples ( $\mathrm{p} \leq 0.05)$.

Results / Conclusion: A total of 451 adult patients were implanted and was necessary to disconnect electrodes in 35 cases. The main causes that led to the need to disconnect electrodes were: non-auditory stimulation, electrode failure / malfunction and incomplete insertion of the electrode array.Some of these situations may overlap.All patients were evaluated with the battery of tests developed and used in our Department to monitor functional benefit in implanted patients. This battery includes the tests: monosyllabic word test, numbers test, sentences test, disyllables word test and consonants test.The results revealed statistical difference in testing the monosyllables and phrases test. The patients were also grouped according to the number of electrodes disconnected and the battery of tests performed. A variation in the results according to the number of electrodes turned off was present, although it was not statistically significant.Regarding the consequences, our sample showed a decrease in auditory performance in some cases. However, with adequate programming, this decrease does not seem to compromise the overall auditory performance of the cochlear implant, not justifying its replacement.

Keywords: Cochlear Implant; Eletrodes; Switch off; Disconnect

\section{Introduction}

In 1961 Dr. William House performed the first single-channel cochlear implant [1]. From that date until today, technological evolution has allowed tremendous advances in the evolution of Cochlear Implant (CI), implantation technique and indications.
The evolution of the CI has been notable in the development of the external component - microphone, speech processor, transmitter - with a constant evolution over the years. This same evolution allows us to have speech processors miniaturized and increasingly effective in auditory processing. The inner component is formed by the receiver and the electrode array. The receptor has remained similar over the years, but the electrode array has evolved considerably and we now have several types of electrode bundles available for cochlear implantation [2].

This evolution allowed to extend the surgical indication to patients with anatomical alterations of the cochlea and other associated clinical conditions that previously contraindicated the surgery. Cochlear implantation has thus become the most widely used and consensual therapeutic method in the treatment of severe to profound neurosensory deafness.

The widening of clinical indications for cochlear implantation increased not only the number of patients implanted but also the number of new difficulties and complications associated with implantation $[3,4]$.

One commonly used division to characterize complications is the separation of major and minor complications. In the group of major complications we have meningitis, immediate postoperative facial paralysis, implant failure, flap dehiscence, surgical removal necessity. In the minor complications we have wound infection, vestibular symptoms, non-immediate facial paralysis and non-auditory electrical stimulation $[5,6]$.

\section{Objectives}

To evaluate the different causes that led to the need to disconnect electrodes in the patients implanted in the Functional Unit of Cochlear Implants and to verify if there were alterations in the auditory performance comparing with the patients in whom it was not necessary to disconnect electrodes.

\section{Methods}

Review of all cases of adult patients implanted in the Unit for Cochlear Implantation in Coimbra Hospital and University Center 
in which was necessary to swicth off electrodes, their causes and consequences in terms of hearing performance. We used the SPSS 23, with T-test for paired samples ( $\mathrm{p} \leq 0.05)$.

\section{Results and Discussion}

From 1985 to September 2016, 457 adult patients were implanted, and in 35 cases, electrodes had to be disconnected. Of these $49.2 \%$ are women and $50.8 \%$ are men. The causes of deafness found were $25 \%$ traumatic brain injury, $25 \%$ otosclerosis, $25 \%$ progressive sensorineural deafness, $16.7 \%$ congenital unidentified and $8.3 \%$ Meniere's disease. These data corroborate that described in the literature. The Cochlear implant model was used in 276 cases (60.39\%), Med-El in 180 cases (39.39\%) and in only one case (0.22\%) Advance Bionics. In the intraoperative period, the impedances showed $89.7 \%$ of cases without alterations, 3.5\% of impedances could not be measured (old models) and in $6.8 \%$ of the cases, changes were found in the electrodes.

The main causes that led to the need to disconnect electrodes were: non-auditory stimulation, electrode failure / malfunction and incomplete insertion of the electrode array.

Non-auditory stimulation was found in 9 cases. Within the non-auditory stimulation we had 5 cases of facial nerve stimulation and 4 cases of craniofacial pain (Table 1). Nonauditory stimulation, especially facial nerve stimulation, is more frequent in patients with cochlear malformations, otosclerosis and cochlear ossification (postmeningitis and oto-syphilis). This complication can occur when the implant is first activated or after 10 years of implantation but most cases have manifestation in the first year $[7,8]$. In our analysis, patients developed this complication in all cases at the time of first cochlear implant connection.

The electrodes may come into electrical fault with open circuit or short circuit. Open circuit is described by high impedances $(>$ 20-30 kilohms) and short circuit by low impedance ( $<1$ kilohm) [9].

In our series we had 11 patients with open circuit electrodes (31.4\%) and 12 patients with short circuit electrodes (34.3\%) (Table 1).

Incomplete insertion is expectable in a cochlea with malformations but occasionally it may also occur in patients with normal cochlear anatomy. The main factors related to the insertion of the electrode array that can led to changes in its insertion and auditory performance are 3. (1) The anatomy of the cochlea, which in itself may not allow the introduction of the electrode array; (2) Surgical insertion is also an important factor. The concept of soft surgery is increasingly used given the recognition of trauma to the inner ear by the insertion of the electrode array during surgery. From small displacements of the basilar membrane to the fracture of the bone spiral lamina that can lead the displacement of the electrode array from the tympanic ramp to the vestibular ramp. With the advancement of imaging techniques, recent studies have shown that this event of displacement of the electrode array from the tympanic to the vestibular ramp is more frequent than thought and may be a reason for less favorable auditory performance in several patients. The cochleae do not all have the same shape and dimension whereby the (3) electrode array chosen for an implanted patient should be considered in an individualized manner $[10,11,12]$.

Of these 35 patients only 3 patients were left with electrodes outside the cochlea ( $8.6 \%$ ) (Table 1 ). In this 3 cases the cochlear anatomy was normal, however, the complete introduction of the electrode array during surgery was not possible.

The statistical analysis performed in the comparison of groups is presented below. The groups were evaluated with the battery of tests developed and used in the CHUC ORL Service to monitor functional benefit in implanted patients. This battery includes the tests: monosyllabic word test, numbers test, sentences test, disyllables word test and consonants test [13].

Initially a division in two groups was made. In one group the patients implanted with electrodes switched off and in the other one the patients without electrodes switched off. In the evaluation of auditory sensibility with implants (tonal benefit) in the free field, it was verified that there was a statistically significant difference in all frequencies with the exception of 500 $\mathrm{Hz}$ (Figure 1). In the evaluation of the discrimination of free-field disyllables word test it was verified that there is only statistical difference in the intensities of 70 and $80 \mathrm{~dB}$ SPL (Figure 2). In the evaluation with the monosyllables, numbers, phrases, 100 words and consonants test there is a statistically significant difference only in the monosyllables and phrases test (Figure 3).

This battery of tests was also compared among patients with the different causes of electrode disconnection. We created 4 patient groups (1) with short circuit electrodes, (2) open circuit, (3) with electrodes outside the cochlea and (4) with non-auditory stimulation. In Graphic 1 we can observe that there are variations in the results according to the type of failure, although this is not statistically significant.

In the 35 cases described it was necessary to switch off 1 electrode in 20 cases, 2 electrodes in 7 cases, 3 electrodes in 5 cases and 4 electrodes in 3 cases (Table 2). We also compare our battery of tests for these 4 groups of patients (Graphic 2). We can observe that there is variation in the results according to

Table 1: Distribution of individuals according to the causes for IC electrode disconnection OP_SC ${ }^{\mathbf{a}}$

\begin{tabular}{|c|c|c|c|c|}
\hline & Frequency & Percent & $\begin{array}{c}\text { Valid } \\
\text { Percent }\end{array}$ & $\begin{array}{c}\text { Cumulative } \\
\text { Percent }\end{array}$ \\
\hline $\begin{array}{l}\text { Valid Open } \\
\text { circuit }\end{array}$ & 11 & 31,4 & 31,4 & 31,4 \\
\hline Short circuit & 12 & 34,3 & 34,3 & 65,7 \\
\hline Out of cochlea & 3 & 8,6 & 8,6 & 74,3 \\
\hline $\begin{array}{l}\text { Pain/facial } \\
\text { stimulation }\end{array}$ & 9 & 25,7 & 25,7 & 100,0 \\
\hline Total & 35 & 100,0 & 100,0 & \\
\hline
\end{tabular}


the number of electrodes turned off, although it is not statistically significant.

\section{Conclusion}

The causes that require the disconnection of electrodes in cochlear implants are well identified and studied: non-auditory stimulation; Failure / malfunction of electrodes; Incomplete insertion of the electrode array. Regarding the consequences,

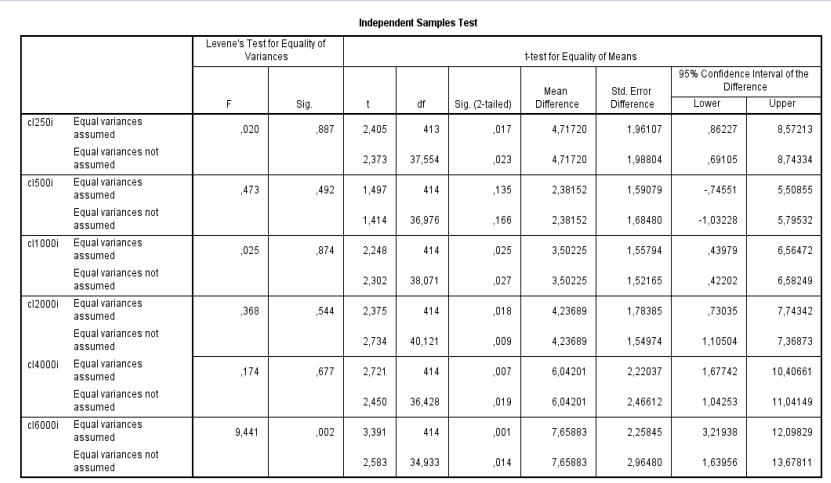

Figure 1: Comparison of the two groups regarding auditory sensitivity with $\mathrm{CI}$ in free field.

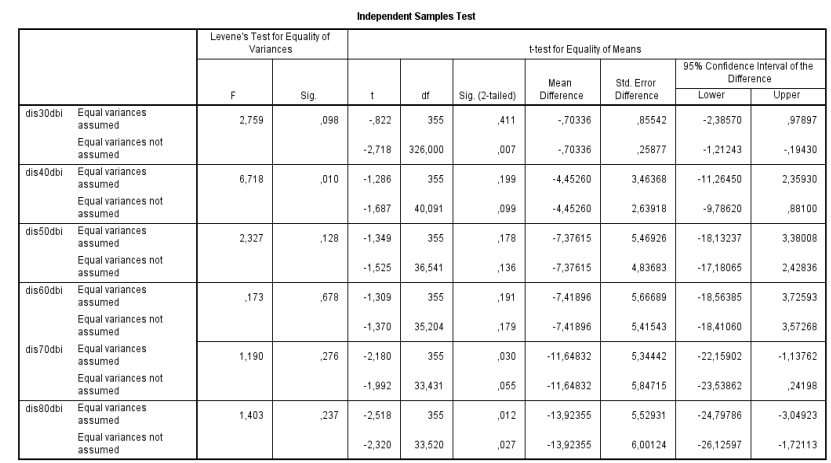

Figure 2: Comparison of the results of disyllabic test with $\mathrm{CI}$ in free field.

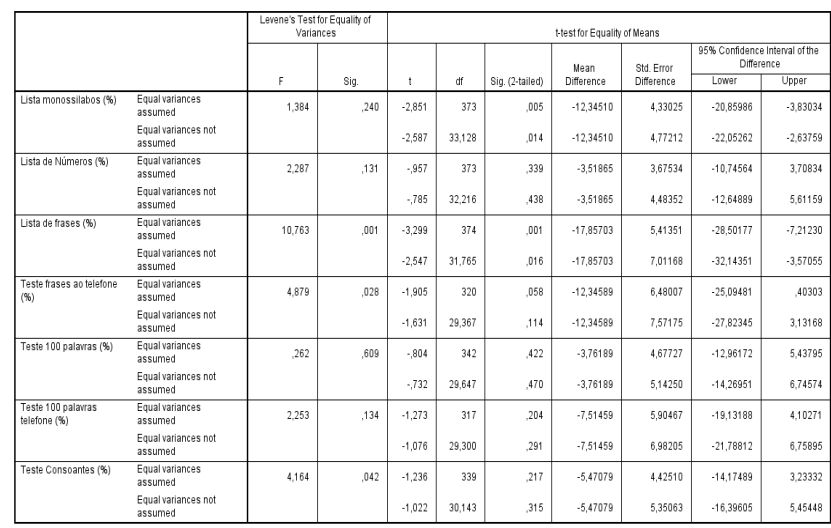

Figure 3: Comparison of the two groups using the results in the performance in the different tests applied.

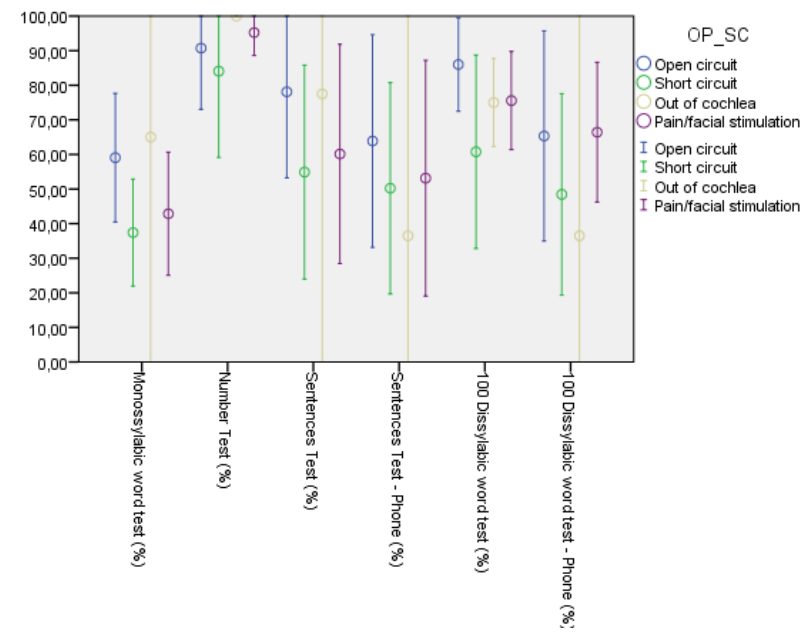

Graphic 1: Comparison of performance in the different tests applied according to the type of anomaly of the electrode array.

Table 2 - Distribution of individuals according to the number of electrodes disconnected.

N_elect_off ${ }^{a}$

\begin{tabular}{|c|c|c|c|c|}
\hline & Frequency & Percent & $\begin{array}{c}\text { Valid } \\
\text { Percent }\end{array}$ & $\begin{array}{c}\text { Cumulative } \\
\text { Percent }\end{array}$ \\
\hline Valid 1 & 20 & 57,1 & 57,1 & 57,1 \\
\hline 2 & 7 & 20,0 & 20,0 & 77,1 \\
\hline 3 & 5 & 14,3 & 14,3 & 91,4 \\
\hline 4 & 3 & 8,6 & 8,6 & 100,0 \\
\hline Total & 35 & 100,0 & 100,0 & \\
\hline \multicolumn{2}{|r|}{ a. Electr_deslig = Yes } \\
\end{tabular}

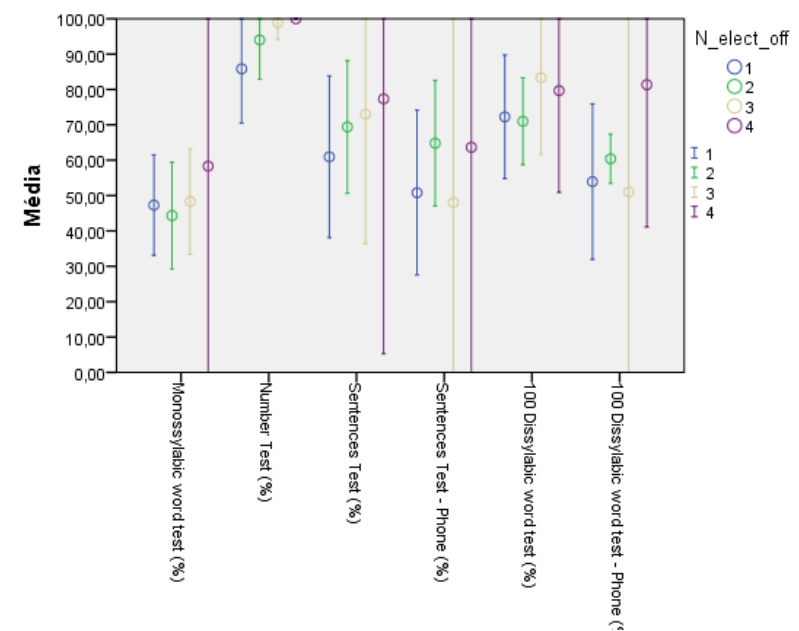

Graphic 2: Comparison of performance in the different tests applied according to the number of electrodes off.

our sample showed a decrease in auditory performance in some cases. However, with adequate programming, this decrease does not seem to compromise the overall auditory performance of the cochlear implant, not justifying its replacement. 


\section{References}

1. Berliner KI, House WF. The cochlear implant program: an overview. Ann Otol Rhinol Laryngol Suppl. 1982;91(2 Pt 3):11-14.

2. Hainarosie $M$, Zainea V, Hainarosie R. The evolution of cochlear implant technology and its clinical relevance. J Med Life. 2014;7(Spec Iss 2):1-4.

3. Parisier SC, Chute PM, Popp AL. Cochlear implant mechanical failures. Am J Otolaryngol. 1996;17(5):730-734.

4. Kubo T, Massuura S, Iwaki T. Complications of cochlear implant surgery. Oper Tech Otolaryngol Head Neck Surg. 2005;16(2):154-158.

5. David S Chen, Danisa MClarrett, Lingsheng Li, Steve P Bowditch, John K Niparko, Frank RLin. Cochlear Implantation in Older Adults: Longterm Analysis of Complications and Device Survival in a Consecutive Series. OtolNeurotol. 2013;34(7):1272-1277. doi: 10.1097/ MA0.0b013e3182936bb2

6. S. Raghunandhan, Mohan Kameswaran, Anand Kumar RS, Anoop Kumar Agarwal, MohammodDelwar Hossain A Study of Complications and Morbidity Profile in Cochlear Implantation: The MERF Experience. Indian J Otolaryngol Head Neck Surg. 2014;66(Suppl 1):161-168. doi:10.1007/s12070-011-0387-3

7. S Berrettini, A De Vito, L BruSchini, S PASSetti, F ForLi. Facial nerve stimulation after cochlear implantation: our experience. Acta othorinolaryngologica italica. 2011;31(1):11-16.
8. Mohammad Seyyedi, Barbara SHerrmann, Donald KEddington, Joseph BNadol Jr. The Pathologic Basis of Facial Nerve Stimulation in Otosclerosis and Multi-Channel Cochlear Implantation. Otol Neurotol. 2013;34(9): 1603-1609. doi: 10.1097/MA0.0b013e3182979398

9. Jenny L Goehring, Michelle L Hughes, Jacquelyn L Baudhuin, Rodney P Lusk. How well do cochlear implant intraoperative impedance measures predict postoperative electrode function? Otol Neurotol. 2013;34(2):239-244. doi:10.1097/MA0.0b013e31827c9d71

10. Kim S van der Marel, Jeroen J Briaire, Ron Wolterbeek, JorienSnelBongers, Berit M Verbist, Johan HM. Frijns. Diversity in Cochlear Morphology and Its Influence on Cochlear Implant Electrode Position. Ear \& Hear. 2014;35(1):e9-e20. doi: 10.1097/01. aud.0000436256.06395.63

11. George B Wanna, Jack H Noble, Matthew L Carlson, Rene H Gifford, Mary S Dietrich, David SHaynes, et al. Impact of Electrode Design and Surgical Approach on Scalar Location and Cochlear Implant Outcomes. Laryngoscope. 2014;124(Suppl 6):S1-S7. doi: 10.1002/lary.24728

12. Charles C Finley, Margaret W Skinner, Holden TA, Holden LK, Whiting BR, Chole RA, et al. Role of electrode placement as a contributor to variability in cochlear implant outcomes. Otol Neurotol. 2008;29(7):920-928. doi: 10.1097/MA0.0b013e318184f492

13. Jorge Humberto Martins, João Januário, Marisa Alves, Daniela Ramos, Helena Alves, Luís Silva, et al. Validation of monosyllable lists, numbers and sentences for vocal audiometry for adults. Revista Clínica e Pesquisa em Otorrinolaringologia. 2009; 3, number 1. 